\title{
Study of Groove Parameters on the Hydrodynamic Behavior of Spiral-Grooved Thrust Bearing with Gas Lubricant
}

\author{
Xianfei Xia, ${ }^{1,2}$ Yu Chen $\mathbb{D}^{2,3}{ }^{2,3}$ Xiuying Wang, ${ }^{3}$ and Yu Sun ${ }^{2}$ \\ ${ }^{1}$ Nanjing Institute of Agricultural Mechanization, Ministry of Agriculture and Rural Affairs, Nanjing 210014, China \\ ${ }^{2}$ School of Mechanical Engineering, Nanjing University of Science and Technology, Nanjing 210094, China \\ ${ }^{3}$ School of Mechanical Engineering, Jiangsu University of Technology, Changzhou 213001, China
}

Correspondence should be addressed to Yu Chen; chenyunjust@126.com

Received 1 February 2021; Revised 10 March 2021; Accepted 1 April 2021; Published 7 April 2021

Academic Editor: Paolo Pennacchi

Copyright (c) 2021 Xianfei Xia et al. This is an open access article distributed under the Creative Commons Attribution License, which permits unrestricted use, distribution, and reproduction in any medium, provided the original work is properly cited.

The gas-lubricated thrust bearing is widely used in agriculture mechanical systems, and the groove shape plays an important role on the hydrodynamic behavior of spiral-grooved thrust bearing (SGTB). Although the groove shape may change smaller, it is clear that the hydrodynamic response is very sensitive to the groove parameters. This paper proposes a computational method for the analysis of SGTB with gas lubricant, considering the effects of groove parameters. With the compressibility taken into account, the evaluation of lubrication performance for SGTB is obtained by the CFD technology. Also, the simulation results are compared with the published data, which indicates that the presented model of SGTB is able to obtain more realistic results of hydrodynamic characteristics of SGTB. Moreover, the mapping relationship between groove parameter and hydrodynamic behavior of SGTB is represented.

\section{Introduction}

The thrust bearing plays an important role in the agriculture mechanical equipment. During the initial design of product, the lubrication performance of SGTB is often performed considering the different working conditions. However, the groove parameter is a necessary element in the design of SGTB, which will significantly affect the hydrodynamic behavior of SGTB including load carrying capacity, friction torque, and bearing stiffness $[1,2]$. The modeling and analysis of hydrodynamic characteristics of SGTB is a classical problem, and it has received great attention by researchers. Although the variation of groove parameter is smaller, the lubricated film flow would change and the hydrodynamic response could produce the fluctuation. The film flow of gas lubricant is a complex physical phenomenon with the characteristics of compressibility, sensibility, and surface roughness of bearing. Thus, some problems for modeling the hydrodynamic characteristics and lubrication performance analysis of SGTB should be considered $[3,4]$. One of the problems is related to how to describe accurately the hydrodynamic response of SGTB with gas lubricant. The second problem is associated with the evaluation of the hydrodynamic behavior, namely, load carrying capacity, friction torque, and damping coefficient, and the issue of how to obtain the results of SGTB with different groove parameters. The method and techniques of modeling are improved based on the development of faster data processing capabilities for computer. Then, it is necessary to establish the exact model of SGTB for studying the influence of groove parameters on the hydrodynamic behavior of SGTB with gas lubricant $[5,6]$.

Over the last few decades, the hydrodynamic characteristics of thrust bearing based on the theoretical and experimental approach have been studied by many researchers [7-9]. In the early years, Nishio et al. [10] conducted a comparison study for describing the static and dynamic behavior of aerostatic thrust bearing, which was performed by the CFD technology. The numerical results discussed the effects of bearing clearance on the load carrying capacity, static stiffness, and dynamic stiffness. Based on the classical steady-state 2D compressible Reynolds equations, the evaluated model of 
gas-lubricated parallel slider bearing was established by Qiu et al. [11]. This method could carry out the relationship between the texture shape and load carrying capacity. It was well known that the variation of texture geometry and density could cause the change of pressure distribution. In order to explain the effects of temperature on the hydrodynamic response of SGTB with gas lubricant, Zhang et al. [12] introduced the simulation model based on the finite volume method. The simulation results revealed that the temperature played an important role in the hydrodynamic behavior analysis of SGTB. And the issue of how to select a suitable model for lubrication performance evaluation of SGTB was still discussed. Due to the fact that the modeling method is the key element for describing the hydrodynamic behavior of aerostatic bearing, Chang et al. [13] conducted a comparison between Reynolds equation and computational fluid dynamic. The numerical calculation results showed that the Reynolds equations could be solved more quickly and the discharge coefficients as sensitive to the gas film thickness. Ma et al. [14] built the mapping relationship between hydrodynamic response and spiral groove gas face seal in the lowpressure condition. Based on the practical application, the results found that the proposed method explained that higher seal pressure and clearance would provide a larger thermal distortion. Then, Pavel et al. [15] proposed a new simulation method of thrust bearing, which could conduct a fast computation and analysis for transient behavior of thrust bearing. The obtained results illustrated the reason of how to produce the vibration and noise issues of gas turbocharger. According to the Reynolds equations and JFO cavitation model, Hu and Meng [16] conducted a theoretical and experimental investigation for evaluating the transient characteristics of SGTB. In this model, the mass-conserving cavitation was considered and deterministic asperity contacts were given to investigate the tribology characteristics of SGTB. Kim et al. [17] focused on the lubrication performance analysis of thrust bearing by a quasidynamic lubrication model. The Reynolds equations were used to define the lubrication film, and the temperature distribution could be obtained by the energy equation. Then, the lubricated film thickness was measured by the experiment and the error between simulation results and experiment data was only 5\%, which demonstrated the effectiveness of the proposed method.

It is important to note that some researchers have attention to the investigations of the groove shape effects on the hydrodynamic characteristics of thrust bearing [18-20]. The theoretical and experimental study of load carrying capacity for thrust bearing was studied by Fesanghary and Khonsari [21]. In this study, the Reynolds equations were used to represent the load carrying capacity of thrust bearing with different groove shapes. The obtained results was not only a demonstration for the proposed method but also illustrated the influence of groove shape on the load carrying capacity of thrust bearing. For the optimum design problem of thrust bearing, Masayuki et al. [22, 23] established a hydrodynamic analysis model of gas-lubricated thrust bear- ing considering the angular displacement characteristics, and a new groove shape was obtained, which included two curved portions. Wu et al. [24] extended a new CFD model for researching the hydrodynamic response in the grooved two-disc system. Then, the lubricated film flow could be described by the presented model and the effects of groove number were discussed by a numerical example case. Moreover, $\mathrm{Ma}$ and et al. [25] focused on a numerical approach to analyze the thermoelastohydrodynamic characteristics of gas face seal with T-grooves. Based on the analysis results, the thermohydrodynamic effect could cause the decrease of opening force obviously and T-groove gas face seal was more suitable to be employed in the conditions of low pressure and high rotational speed. Considering the effects of surface texturing, Gherca et al. [26] expanded an approach for modeling the lubrication performance analysis of thrust bearing. The operating characteristics of friction torque, film pressure, and film thickness were obtained by the presented method, and the difference in behavior between steady state and transient was represented. With the geometric parameters and operating parameters taken into account, the numerical analysis of mechanical seal was conducted by Sun et al. [27]. The simulation results showed that the increase of pressure of lubricated film, spiral angle, and width ratio of groove could enhance the opening force and leakage rate. Then, the groove parameters played a significant role on the numerical analysis of mechanical seal. Gropper et al. [28] involved a numerical analysis approach for optimization design of thrust bearing. The numerical results illustrated that the surface texture parameters would change the flow state of lubricated medium and the groove parameter analysis was the necessary step for the design of thrust bearing. Furthermore, Lin et al. [29] devoted a methodology to evaluate the influence of groove parameters on the hydrodynamic behavior of SGTB with water lubricant. The cavitation model was introduced in the lubrication model, and the experimental rig was established to measure the stiffness coefficient and damping coefficient of SGTB. The relationship between the geometric properties of groove and lubrication performance was drawn in detail.

It is widely known that gas lubrication plays an important role in the hydrodynamic behavior analysis of SGTB and many researchers concentrated on the modeling and simulation [30, 31]. Thomas et al. [32] established an axisymmetric model of thrust bearing. The inertia terms and compressible fluids at high pressure were considered in the simulation. The experiment results were applied to demonstrate the effectiveness. Wang et al. [33] proposed a calculation method for describing the gas film distribution of spiral groove. The results illustrated that the design of spiral groove was advanced and practicable. Meanwhile, Gao et al. [34] investigated the effects of orifice chamber shapes on the lubrication performance of aerostatic thrust bearing. The results revealed that there were many differences in the performance behavior of aerostatic thrust bearing caused by the different orifice chamber shapes. Considering the steady-state characteristics, Zhang et al. [35] evaluated the lubrication performance of aerostatic bearing. The simulation results represented that lubrication 
performance was more sensitive to the misalignment along the horizontal direction.

In contrast to the extensive study on the hydrodynamic behavior analysis of thrust bearing with different working conditions, devoting to the industrial case study with the effects of groove geometric parameters on the hydrodynamic characteristics of SGTB is more limited. The major emphasis of this paper is investigating the hydrodynamic modeling and analysis of SGTB with gas lubricant. In the simulation, it is significant to find a suitable method for describing the hydrodynamic characteristics of SGTB with different groove parameters. Then, the CFD technology is employed to establish the computational model of SGTB. The precise hydrodynamic behavior of SGTB with different groove parameters can be represented by the method. In order to demonstrate the accuracy of the proposed method, the simulation results are compared with the published data. Finally, the effects of groove parameters on the hydrodynamic response of SGTB are evaluated.

\section{Theoretical Model}

The typical SGTB is diagrammed in Figure 1, which is employed to describe the geometric characteristics between bearing and disc $[36,37]$. It is clearly seen that the groove parameters have an important effect on the gas film feature. Actually, the difference of grooved structure will increase the complexity of gas film flow, and the relative hydrodynamic characteristics of SGTB can be changed. In the schematic of SGTB, $r_{i}$ and $r_{o}$ show the radiuses of inner groove and outer groove, respectively. The spiral angle $(\alpha)$ plays an important role in the lubricant performance of SGTB. The spiral groove line is written as

$$
r=r_{b} e^{\theta \tan \alpha}
$$

where $r_{b}$ and $\theta$ are the base radius of spiral curve and the circumferential coordinate, respectively.

Then, the lubricated film can be defined as

$$
\begin{aligned}
& h=h_{l}, \\
& h=h_{l}+h_{g},
\end{aligned}
$$

where $h_{l}$ is the land depth and $h_{g}$ denotes the grooved depth.

During the calculation of SGTB, the ideal gas film is assumed and the temperature is constant $[38,39]$. The sliding phenomenon is ignored. The continuity equation is expressed as

$$
\frac{\partial \rho}{\partial t}+\nabla \cdot(\rho \vec{v})=0
$$

where $\rho$ and $\vec{v}$ are gas density and velocity vector, respectively. [40]

And the momentum conservation equation is given as

$$
\left\{\begin{array}{l}
\frac{\partial(\rho u)}{\partial t}+\nabla \cdot(\rho u \vec{v})=-\frac{\partial p}{\partial x}+\frac{\partial \tau_{x x}}{\partial x}+\frac{\partial \tau_{y x}}{\partial y}+\frac{\partial \tau_{z x}}{\partial z}+\rho f_{x} \\
\frac{\partial(\rho v)}{\partial t}+\nabla \cdot(\rho v \vec{v})=-\frac{\partial p}{\partial x}+\frac{\partial \tau_{x y}}{\partial x}+\frac{\partial \tau_{y y}}{\partial y}+\frac{\partial \tau_{z y}}{\partial z}+\rho f_{y} \\
\frac{\partial(\rho w)}{\partial t}+\nabla \cdot(\rho w \vec{v})=-\frac{\partial p}{\partial x}+\frac{\partial \tau_{x z}}{\partial x}+\frac{\partial \tau_{y z}}{\partial y}+\frac{\partial \tau_{z z}}{\partial z}+\rho f_{z}
\end{array}\right.
$$

where $p$ is gas pressure, $\tau_{i j}$ denotes the deviator stress component, and $f_{y}$ represents the quality force component. And $u, v$, and $w$ are the velocity components.

In order to evaluate characteristics of SGTB, the load carrying capacity can be obtained as [41]

$$
W=\int_{0}^{2 \pi} \int_{r_{1}}^{r_{2}} p r d r d \theta
$$

The friction is defined as

$$
F=-\int_{0}^{2 \pi} \int_{r_{1}}^{r_{2}} \tau d r d \theta
$$

where $\tau$ is the shear force of lubrication film surface.

The damping coefficient is also an important value on the hydrodynamic behavior of SGTB, and this value is related the load carrying capacity and rotational speed, which can be written as

$$
c=\frac{W\left(h_{g}-\Delta h_{g}\right)-W\left(h_{g}\right)}{\omega \Delta h_{g}},
$$

where $\omega$ is rotational speed and $\Delta h_{g}$ represents slight variation of groove depth. $W\left(h_{g}-\Delta h_{g}\right)$ and $W\left(h_{g}\right)$ are the load carrying capacity at the groove depth of $h_{g}-\Delta h_{g}$ and $h_{g}$, respectively.

To explain the effects of groove parameters on the hydrodynamic behavior of SGTB, the dimensional analysis is introduced for investigating the main parameters, which includes length ratio and width ratio [42]. The length ratio of groove can be written as

$$
\delta_{l}=\frac{r_{o}-r_{i}}{r_{o}-r_{b}}
$$

Then, the width ratio of groove is given as

$$
\delta_{w}=\frac{w_{l}}{w_{g}},
$$

where $w_{g}$ and $w_{l}$ are the lengths of groove and land, respectively. 


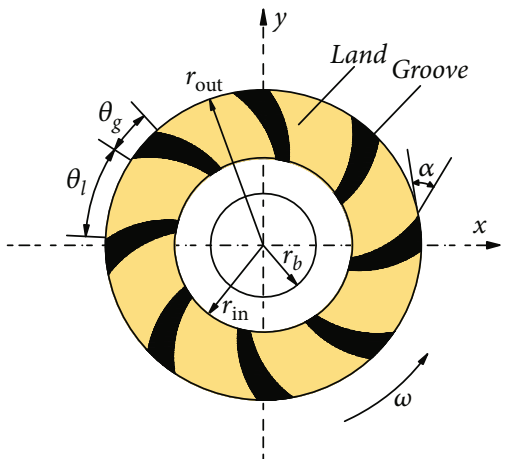

(a)

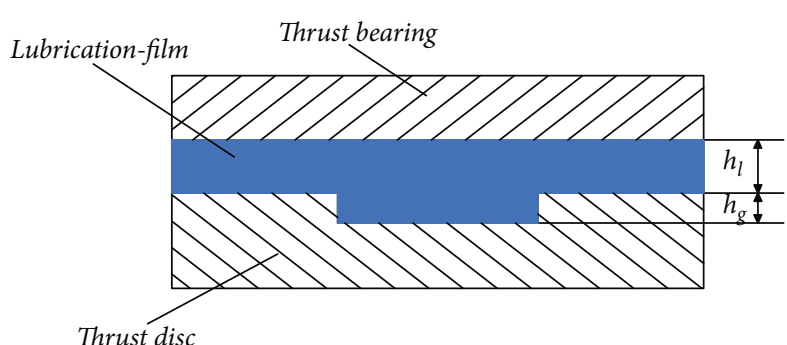

(b)

FIGURE 1: The schematic of spiral-grooved thrust bearing.

\section{Numerical Validation}

In this section, the effectiveness of the proposed method for describing the hydrodynamic characteristics of SGTB is demonstrated. The simulation model is established based on Ref. [43]. The inner radius is $69 \mathrm{~mm}$, the outer radius is $77.78 \mathrm{~mm}$, and the base radius is $58.42 \mathrm{~mm}$. The spiral angle is defined as $15^{\circ}$, and the depths of groove are $0.005 \mathrm{~mm}$. In the first, the convergence and accuracy of solution are sensitive to the equality of mesh model [44]. Fluent is a popular software for conducting the hydrodynamic response of fluid, which includes abundant physical model and advanced numerical methods. Then, the Gambit is a pretreatment software and the higher quality of mesh could be provided for the Fluent, which can ensure the convergence and accuracy of simulation. Fluent and Gambit are employed to study the lubrication performance of SGTB. Based on the characteristics of SGTB, the gas film is defined to the compressible fluid and the pressure-based is employed to the solver type. For the compressible flow, the ideal gas and segregated solver can be used, which has the highly coupled characteristics for velocity, density, and pressure. Then, the second-order upwind is selected and the relaxation factor is selected to 0.3 , which would provide a better convergence. Then, the simulation model is established by the hexahedron element, which could provide a better convergence. It is all known that the quality of mesh can decide the calculation accuracy and the mesh quality of spiral grooved thrust bearing is higher 0.6, as shown in Figure 2(a). In addition, the convergence criteria demand that velocity residual is $10^{-6}$ and the relative difference between mass flow inlet and mass flow outlet is less than $1 \%$. Figure 2(b) shows the effects of oil film layer (element number) on the pressure of SGTB. When the layer is below 10, the error of pressure is larger. However, the deviation value is only $0.9 \%$ between 10 layers and 30 layers. Then, the gas film is divided to 10 layers and the number of elements is 288000 .

In order to evaluate the effectiveness of the proposed model, it is necessary to conduct the hydrodynamic behavior analysis of SGTB and compare with the published results. The model of SGTB in Ref. [43] is used, and the results are shown in Figure 3. According to the results, it is found that the present method could describe the hydrodynamic char- acteristics of SGTB accurately. The gas film pressure increases as the growth of radius, especially in the larger radius condition. Although there is difference between simulation results and experimental data, the maximum deviation is only $5 \%$ and it is accepted in the engineering analysis.

\section{Numerical Results}

4.1. Description of the Spiral-Grooved Thrust Bearing. In this section, the effects of groove parameters on the hydrodynamic characteristics of SGTB are discussed based on the proposed method. The geometric characteristics and simulation parameters of SGTB are given in Table 1. The simulation parameters are selected by Ref. [18], and the temperature is defined as the constant in the simulation. In addition, the simulation results could illustrate how the main parameters (spiral angle, length ratio, and width ratio) affect the lubrication performance of SGTB.

Figure 4 represents the pressure distribution of gas film for SGTB with different groove parameters. The simulation results reveal that the influence of groove parameters on the hydrodynamic characteristics of SGTB is not neglected. The maximum pressure of gas film is $7.517 \times 10^{4} \mathrm{~Pa}, 7.661 \times 10^{4}$ $\mathrm{Pa}$, and $7.188 \times 10^{4} \mathrm{~Pa}$, respectively. It is known that the pressure value plays an important role on the load carrying capacity of SGTB. With different groove parameters, the load carrying capacity is $51.45 \mathrm{~N}, 51.8 \mathrm{~N}$, and $49.44 \mathrm{~N}$, respectively. The changed trend of load carrying capacity is similar to this of pressure value. Meanwhile, the results of gas film stiffness are compared. The values of gas film stiffness are $1.038 \times 10^{7} \mathrm{~N} / \mathrm{m}, \quad 9.41 \times 10^{6} \mathrm{~N} / \mathrm{m}$, and $1.061 \times 10^{7} \mathrm{~N} / \mathrm{m}$. Then, the simulation results illustrate that the investigation of groove parameters effects should be conducted.

4.2. Influence of Spiral Angle. This section investigates how the spiral angle influences the hydrodynamic characteristics of SGTB whereby the groove length ratio and groove width ratio are defined as the constant value equals to 0.8 in the simulation. The rotational speeds of shaft are chosen to be $20000 \mathrm{rpm}, 40000 \mathrm{rpm}, 60000 \mathrm{rpm}$, and $80000 \mathrm{rpm}$, respectively. Then, the simulation results are shown in Figure 5.

From the perspective of the design, the well hydrodynamic characteristics and high lubrication performance are 


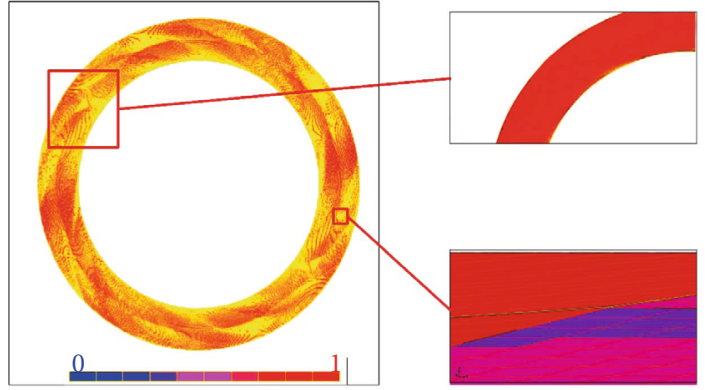

(a)

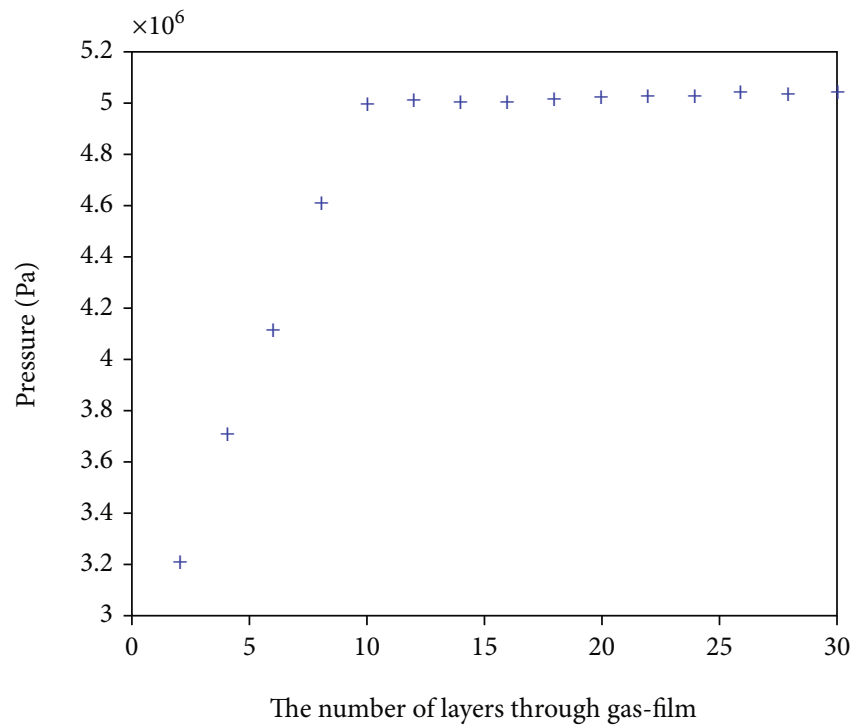

(b)

FIgURE 2: The mesh model and mesh sensitivity of spiral-grooved thrust bearing.

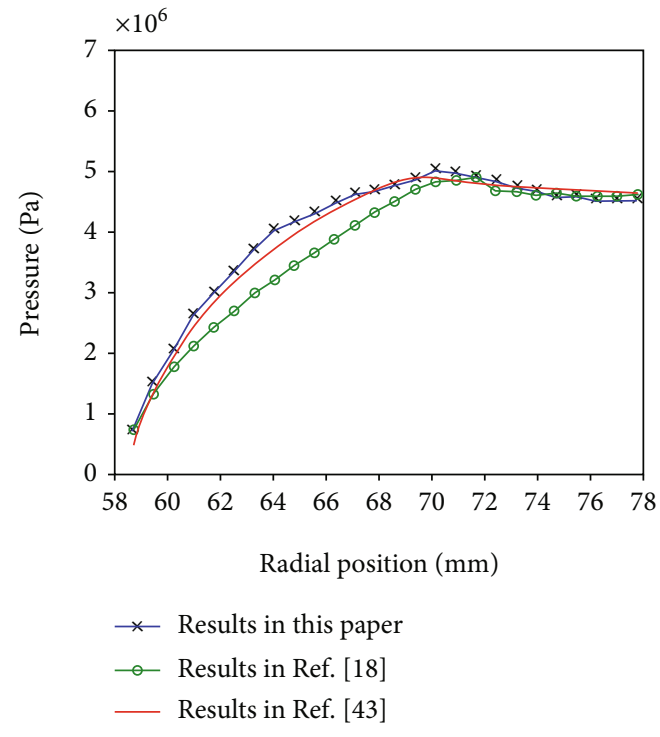

Figure 3: Comparison between the present model and experiment data.

expected. The maximum pressure value is employed to reveal the pressure distribution of gas film, and it is closely related to the load carrying capacity of SGTB. As shown in Figure 5(a), when the spiral angle increases from $15^{\circ}$ to $30^{\circ}$, the peak value of maximum pressure appears to the spiral angle that reaches to $20^{\circ}$, particularly at a higher rotational speed. From the simulation results, it is also known that the various trend of load carrying capacity is similar with the path of maximum pressure. The reason of this phenomenon can be concluded that the pressure value and load section play the important roles in the load carrying capacity. A higher pressure value will present a larger load carrying capacity. However, the pressure value of gas film is more sensitive to the value of spiral angle. Thus, in order to ensure the effects of spiral angle on the lubrication performance of SGTB, the hydrodynamic characteristics should be performed with respect to spiral angle and rotational speed.

In order to illustrate the influence of spiral angle on the friction torque of SGTB, the cases are conducted based on the presented model. Figure 5(c) shows that the simulation results of friction torque are evaluated with consideration of different spiral angles and rotational speeds. When the spiral angle is $20^{\circ}$ and the rotational speed is $20000 \mathrm{rpm}$, the deviation value of friction torque is $1.62 \times 10^{-3} \mathrm{Nm}$. As the rotational speed reaches $80000 \mathrm{rpm}$, this value increases to $6.699 \times 10^{-3} \mathrm{Nm}$. It is obtained that a higher rotational and a bigger spiral angle will cause a larger friction torque. Then, Figure 5(d) shows that the friction loss of SGTB is enlarged by the increase of spiral angle and rotational speed, particularly the higher rotational speed. It is clearly illustrated that the effects of spiral angle on the friction loss become strong at a higher speed due to the increase of mechanical system energy. The reason of this phenomenon can be illustrated that the flow field intensity changes as the increase of spiral angle and rotational speed, which could be the decrease of velocity gradient gas and friction force. Therefore, the analysis of friction torque and friction loss should be conducted during the design of SGTB.

According to the plot in Figure 5(e), the effects of spiral angle on the bearing stiffness are employed to evaluate the resistance capability of deformation for SGTB. It is seen that the increase of spiral angle will decrease the bearing stiffness. However, the value of gas film stiffness increases as the growth of rotational speed. The reason of this phenomenon lies in the fact that the variation of groove geometric model will cause difference of gas film pressure distribution. Moreover, the damping coefficient of SGTB is displayed in Figure 5(f), which can reflect that gas film stiffness and 
TABLE 1: Simulation parameters of the spiral-grooved thrust bearing.

\begin{tabular}{lccc}
\hline Parameters & Value & Parameters & Value \\
\hline Based radius $r_{b}(\mathrm{~mm})$ & 12.5 & Inner radius $r_{\text {in }}(\mathrm{mm})$ & $13.75(0.6), 15.6(0.7), 16.25(0.8), 7.5(0.9)$ \\
Outer radius $r_{\text {out }}(\mathrm{mm})$ & 25 & Spiral angle $\alpha\left(^{\circ}\right)$ & $15,20,25,30$ \\
Groove number $(n)$ & 16 & Rotational speed $\omega(\mathrm{rpm})$ & $20000,40000,60000,80000$ \\
Land depth $h_{l}(\mathrm{~mm})$ & 0.011 & Groove width ratio & $0.6,0.8,1,1.2$ \\
Groove depth $h_{g}(\mathrm{~mm})$ & 0.033 & Gas viscosity $v(\mathrm{mPa} \cdot \mathrm{s})$ & 0.0185 \\
Temperature $\left({ }^{\circ} \mathrm{C}\right)$ & 20 & Thermal conductivity $(\mathrm{W} / \mathrm{mK})$ & 0.0256 \\
\hline
\end{tabular}

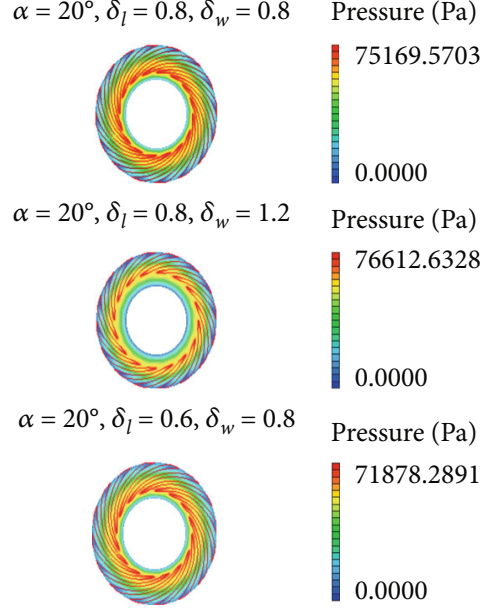

FIGURE 4: The pressure distribution of spiral-grooved thrust bearing with different groove parameters.

rotational speed play the important roles on the damping coefficient. Evidently, as the spiral angle and rotational speed increase, the damping coefficient decreases. In other words, the damping coefficient is independent of the pressure at equilibrium position.

4.3. Influence of Groove Length Ratio. The intention of this study is to explore the relationship between groove length ratio and hydrodynamic behavior of SGTB. The initial condition assumes that the spiral angle is $20^{\circ}$ and the groove length ratio is 0.8 . The rotational speeds are $20000 \mathrm{rpm}, 40000 \mathrm{rpm}$, $60000 \mathrm{rpm}$, and $80000 \mathrm{rpm}$, respectively. It is important to note that the results of hydrodynamic simulation for the SGTB are shown after the value is convergence.

Firstly, in this section, the effects of groove length ratio on the hydrodynamic characteristics of SGTB are analyzed. In the simulation results, the coupling effects of groove length ratio and rotational speed are acquired and plotted in Figure 6. Figures 6(a) and 6(b) represent the maximum pressure and load carrying capacity of SGTB. Compared with the trajectory of SGTB with different spiral angles, the characteristics of these values have a difference. It is clear to see that the value of maximum pressure keeps a stable value when the groove length ratio is lower than 0.7. However, the value of maximum pressure decreases obviously as the increase of groove length ratio. Although the deviation value is not greatly affected at a lower rotational speed, this value is still changed, which may decrease the load carrying capacity. Meanwhile, it also represents the relationship between pressure value and load carrying capacity. And the hydrodynamic response analysis of SGTB with different groove length ratios should be conducted during the design.

To figure out the influence of groove length ratio on the hydrodynamic response of SGTB, different comparative models (SGTB with different groove length ratios) are established, as shown in Figures 6(c) and 6(d). Apparently, the increase of groove length ratio leads to the decrease of friction torque and friction loss slowly. Due to transformation of geometric parameter, the state of gas flow has a difference at every section, and the friction force is also changed. In addition, although the deviation values of friction torque and friction loss are small, this phenomenon is not neglected and the operation efficiency of SGTB will come down.

As mentioned before, the gas film stiffness plays the crucial role on the hydrodynamic behavior of SGTB. With the growth of groove length ratio, the gas film stiffness increases gradually, as shown in Figure 6(e). However, when the groove length ratio exceeds 0.8 , the value of gas film stiffness decreases suddenly. During the design of SGTB, the designer should conduct a perfect investigation of lubricant performance and the mapping relationship between groove geometric parameters and gas film stiffness. Figure 6(f) indicates the variations of damping coefficient with respect to groove length ratio at different rotational speeds. It is important to note that the various groove structural parameters can affect the flow state of gas and then the pressure distribution, density, and flow direction of gas film would change. The value of damping coefficient is related with the load carrying capacity of gas film and rotational speed. The value can represent the ability of opposed load variation for gas film, and it is decided by the pressure and thickness of gas film. When the groove length ratio is 0.8 , the damping coefficient reaches to the peak value by the simulation results. Then, a reasonable structural parameter of spiral groove can contribute to a higher lubrication performance of SGTB. When the groove length ratio is 0.8 , the damping coefficient reaches to the peak value by the simulation results. And the minimum value that appears under the groove length ratio is 0.9. Meanwhile, the value of damping coefficient decreases as the increase of rotational speed. Then, a reasonable structural parameter of spiral groove can contribute to a higher lubrication performance of SGTB. 


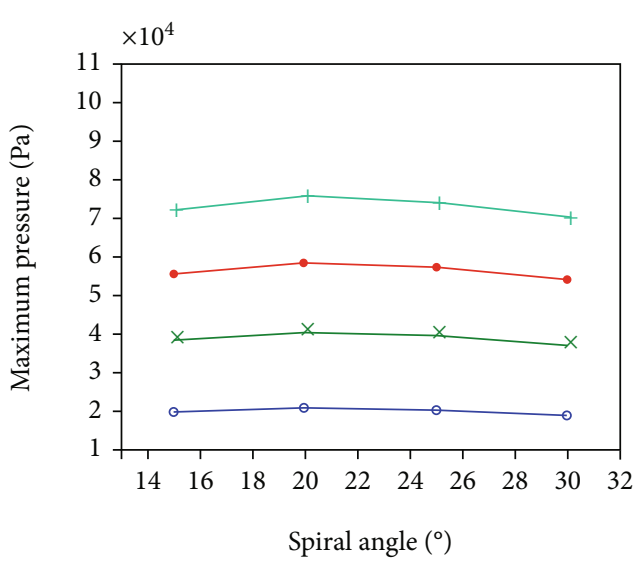

(a)

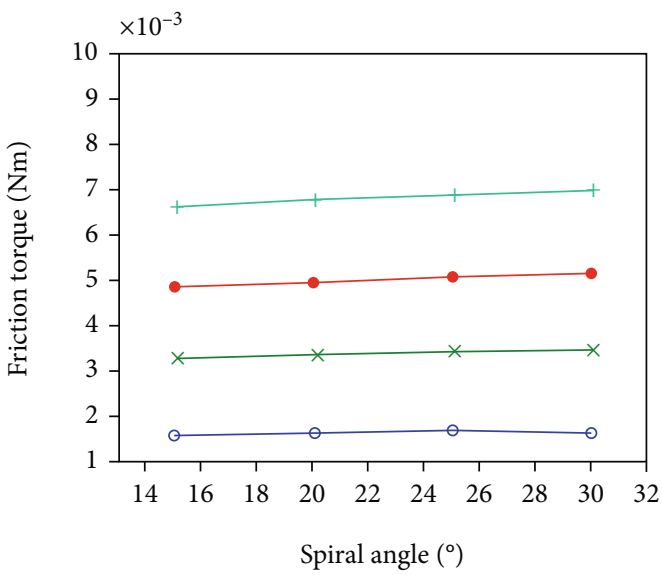

(c)

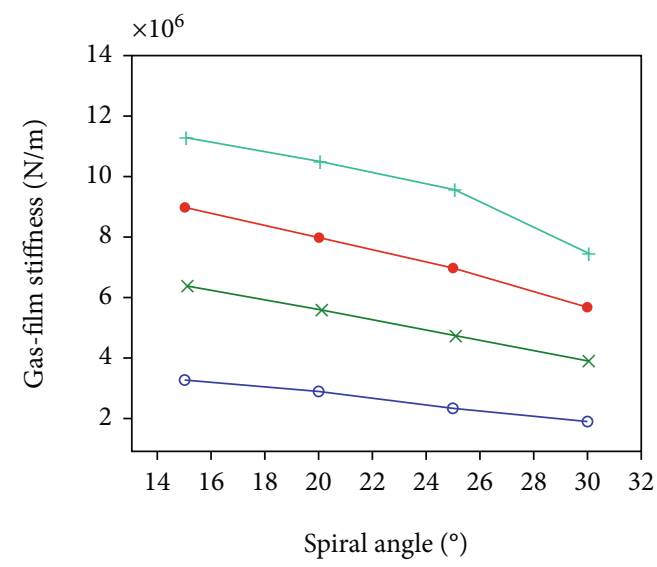

(e)

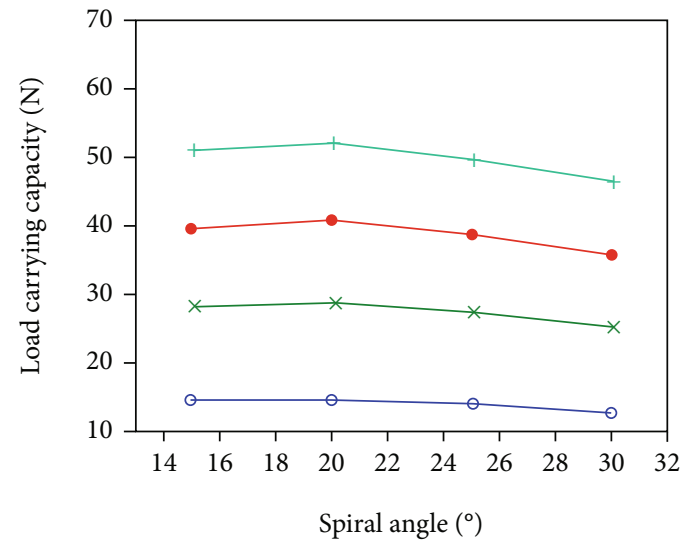

(b)

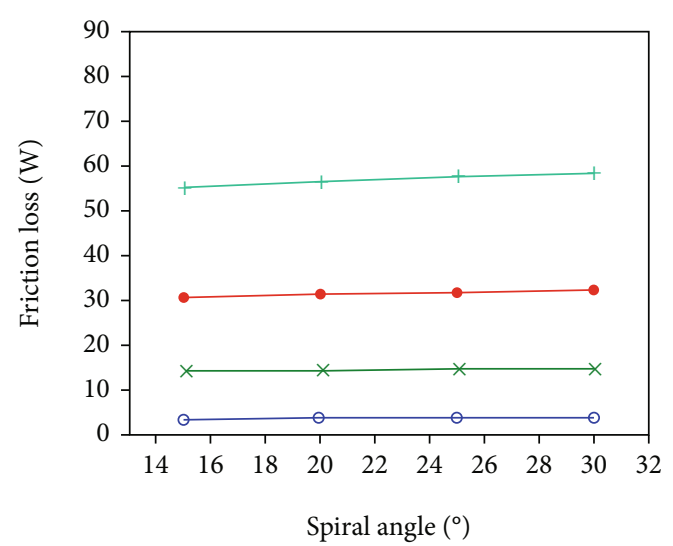

(d)

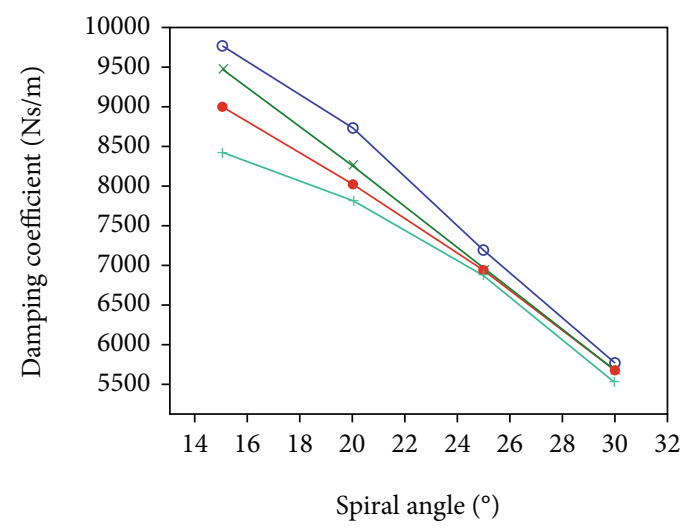

$\begin{aligned} \odot n=20000 \mathrm{rpm}-n & -60000 \mathrm{rpm} \\ \times n & =40000 \mathrm{rpm}+n=80000 \mathrm{rpm}\end{aligned}$

(f)

FiguRE 5: The lubrication performance of spiral-grooved thrust bearing with different spiral angles: (a) maximum pressure, (b) load carry capacity, (c) friction torque, (d) friction loss, (e) gas film stiffness, and (f) damping coefficient.

4.4. Influence of Groove Width Ratio. In this section, the hydrodynamic behavior of SGTB with different groove width ratios is discussed based on the proposed method. To obtain the influence of groove width ratio on the lubrication performance of SGTB, the rotational speed is also defined as a variable. In the simulation model, the spiral angle and groove length ratio are $20^{\circ}$ and 0.8 , respectively. And the values of rotational speed are $20000 \mathrm{rpm}, 40000 \mathrm{rpm}, 60000 \mathrm{rpm}$, and $80000 \mathrm{rpm}$.

Figure 7(a) illustrates the maximum pressure of gas film considering the effects of groove length ratio. When the groove length ratio increases from 0.6 to 1.2 , the maximum 


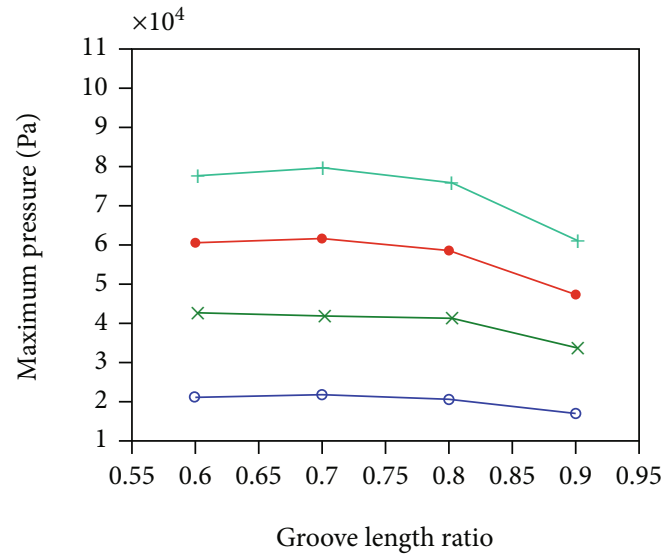

(a)

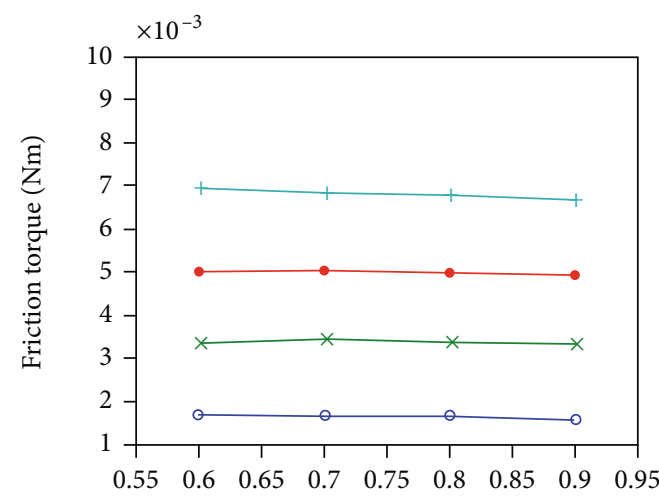

Groove length ratio

(c)

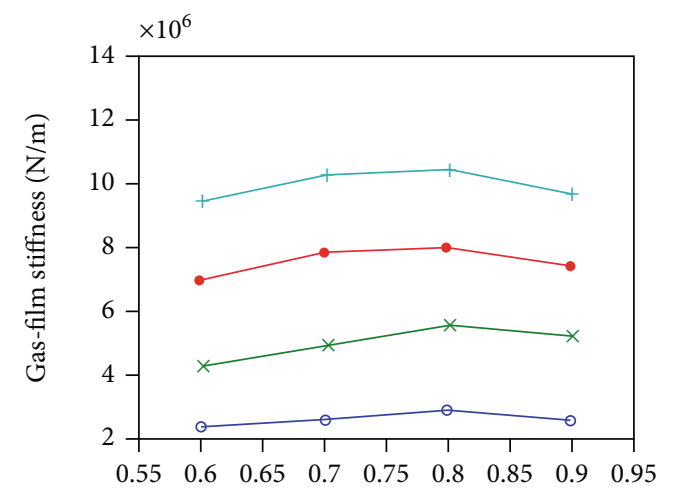

Groove length ratio

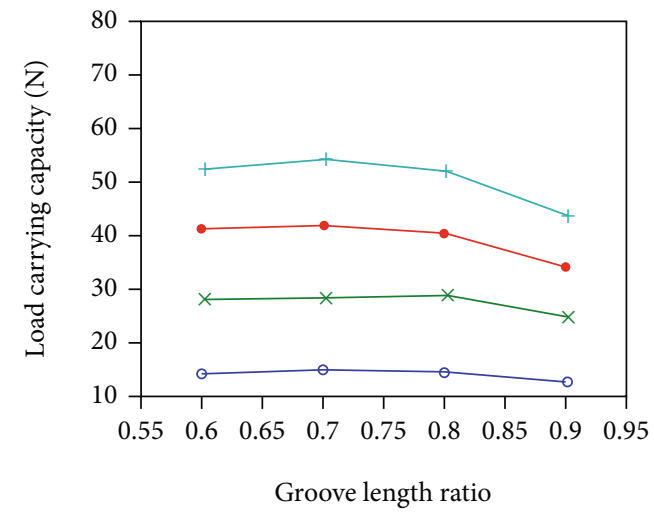

(b)

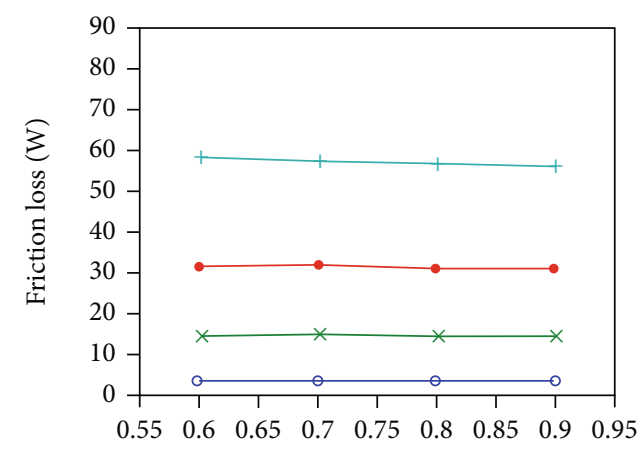

Groove length ratio

(d)

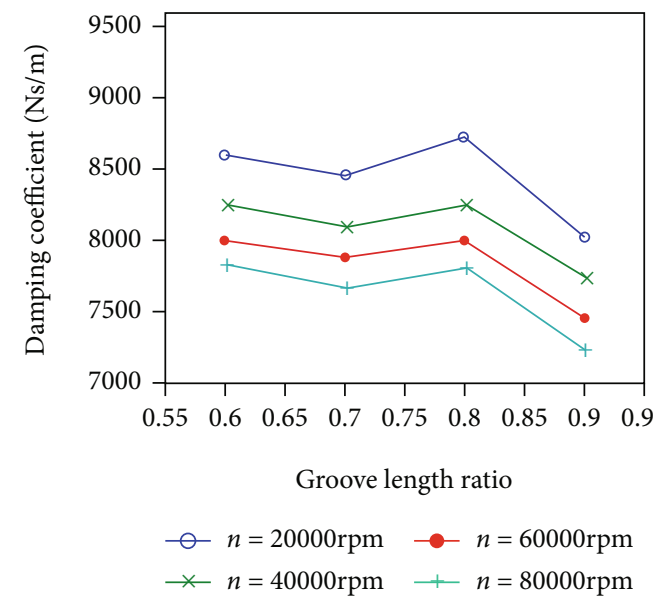

(f)

FIgURE 6: The lubrication performance of spiral-grooved thrust bearing with different groove length ratios: (a) maximum pressure, (b) load carrying capacity, (c) friction torque, (d) friction loss, (e) gas film stiffness, and (f) damping coefficient.

pressure of gas film decreases from $7.952 \times 10^{4} \mathrm{~Pa}$ to 7.188 $\times 10^{4} \mathrm{~Pa}$ at $80000 \mathrm{rpm}$. The maximum pressure of gas film for the SGTB with a lower groove width ratio is larger than that of a larger groove width ratio. Moreover, the maximum pressure of gas film increases as the growth of rotational speed. Combined with the influence of groove width ratio, the variation of maximum pressure of gas film is obvious at a higher rotational speed. That is to say, the groove width ratio makes the maximum pressure of gas film decrease especially under the high rotational speed condition. Generally, in the lower gas film pressure, the load carrying capacity is smaller, as shown in Figure $7(\mathrm{~b})$. The simulation results 


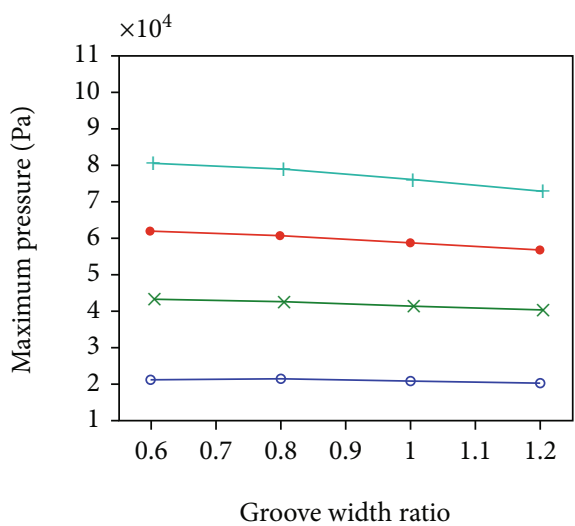

(a)

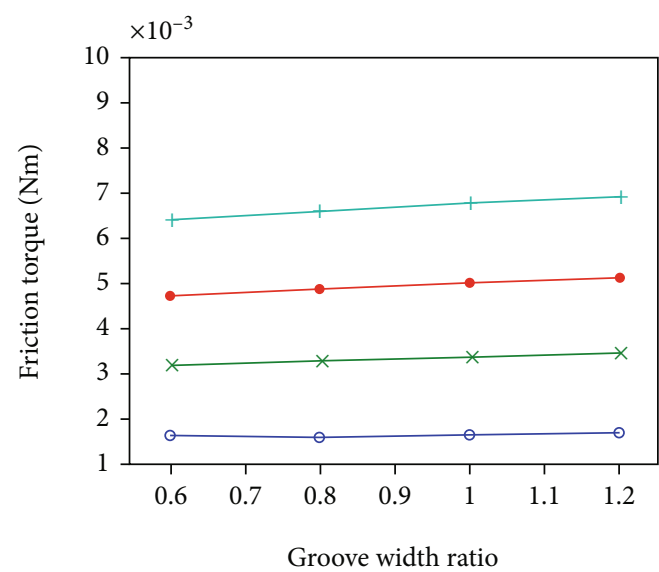

(c)

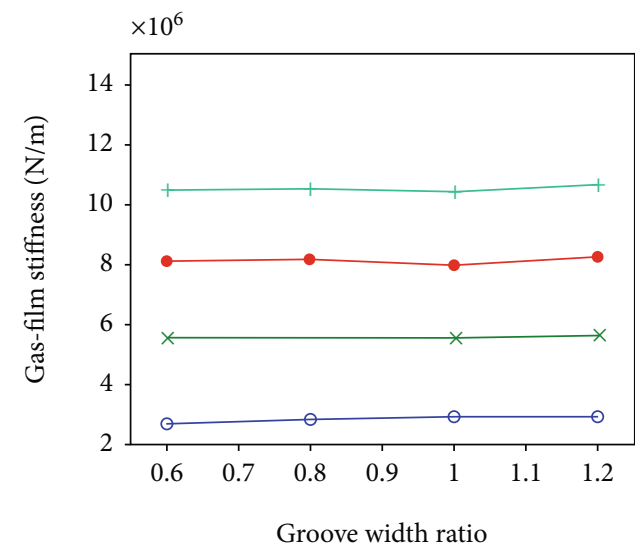

(e)

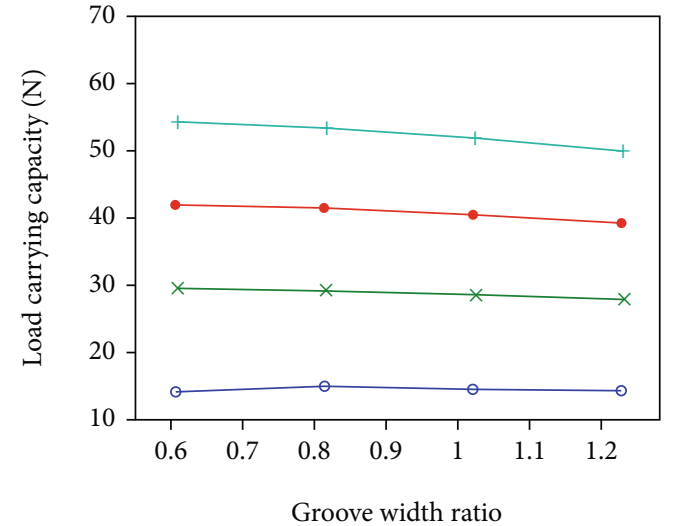

(b)

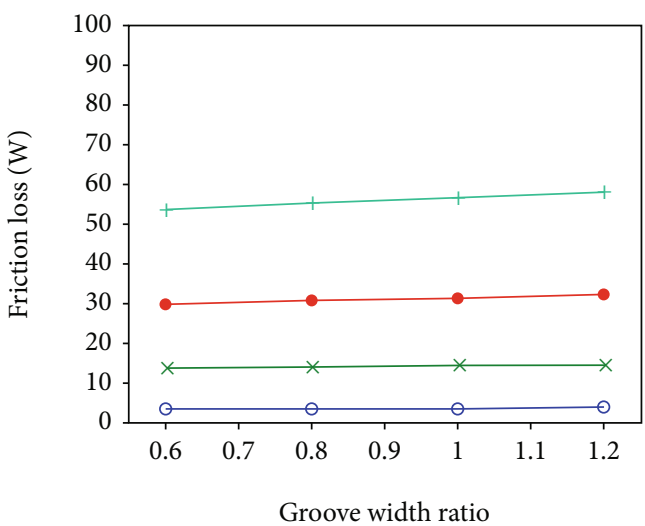

(d)

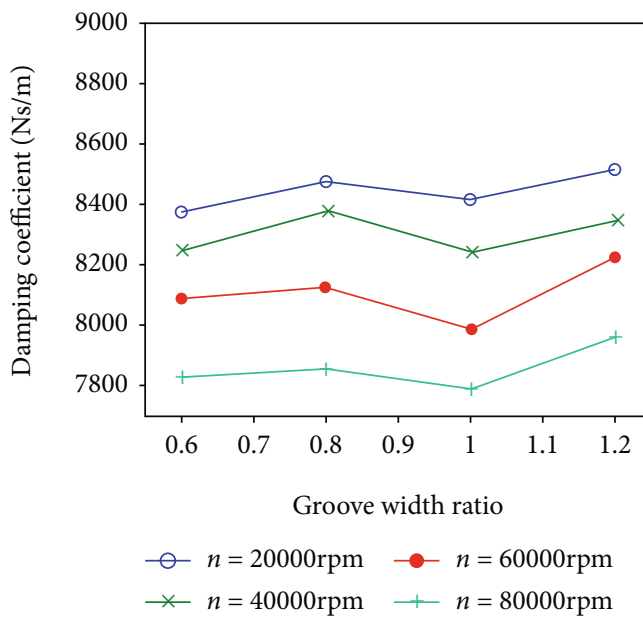

(f)

FiguRE 7: The lubrication performance of spiral-grooved thrust bearing with different groove width ratios: (a) maximum pressure, (b) load carrying capacity, (c) friction torque, (d) friction loss, (e) gas film stiffness, and (f) damping coefficient.

illustrate that the change of pressure value leads to significant effects of load carrying capacity. Then, the groove width ratio plays an important role on the hydrodynamic response of SGTB.

The friction torque of SGTB with different groove width ratios is shown in Figure $7(\mathrm{c})$. When the groove width ratio is 0.6 , the friction torque is $1.589 \times 10^{-3} \mathrm{Nm}$ at the rotational speed $20000 \mathrm{rpm}$. While the groove width ratio is 1.2 , the friction torque increases to $1.656 \times 10^{-3} \mathrm{Nm}$, which is quite different of the effects of groove length ratio. And also, the friction torque of SGTB at a lower rotational speed is much smaller than that at a higher rotational speed. Then, the 
effects of groove width ratio and rotational speed on the friction loss are listed in Figure 7(d). Apparently, the influence of rotational speed is obvious, which makes the friction loss increases especially at a higher rotational speed. According to the simulation results, we can also see that the increase of friction loss is more gently as the growth of groove width ratio. For example, when the groove width ratio is 0.6 , the friction loss of SGTB is $3.328 \mathrm{~W}$ at $80000 \mathrm{rpm}$. As the groove width ratio increases to 1.2 , the value of friction loss is $3.468 \mathrm{~W}$.

Figures 7(e) and 7(f) discuss the effects of groove width ratio on the gas film stiffness and damping coefficient. It can be seen that the groove width ratio affects the damping coefficient obviously, compared with the gas film stiffness. As the groove width ratio increases, the gas film stiffness increases slowly. When the groove width ratio is 0.6 and rotational speed is $20000 \mathrm{rpm}$, the gas film stiffness is $2.691 \times$ $10^{6} \mathrm{~N} / \mathrm{m}$. As the groove width ratio gets to 1.2 , the value of gas film stiffness is $2.905 \times 10^{6} \mathrm{~N} / \mathrm{m}$ and the increased percentage is only $7.95 \%$. However, the fluctuation phenomenon of damping coefficient appears under the different groove width ratio. When the groove width ratio is 1 , the inflection point of damping coefficient occurs during the groove width ratio. Therefore, the relationship between lubrication performance of SGTB and groove geometric parameters should considered in the design.

\section{Conclusions}

In this work, a comprehensive method to evaluate the hydrodynamic characteristics of SGTB with gas lubricant is presented. The numerical investigations are implemented as a general example to explain the method and procedures adopted throughout this work. For this objective, the simulation results are compared with the published data, which demonstrates the effectiveness of the proposed model. Meanwhile, the results also represent that the presented method could provide a fast and accurate way for investigating the hydrodynamic behavior of SGTB. The introduction of CFD technology is to develop the universal simulation method, which can achieve structural geometrical model. With the groove geometric parameters taken into account, the lubrication performance analysis of SGTB is performed. The simulation results reveal that the groove plays an important role in the lubrication performance analysis of SGTB. In particular, compared with the rotational speed condition, the variation of hydrodynamic characteristics is more sensitive. In addition, the investigation of groove parameter on the hydrodynamic behavior of SGTB makes sense to engineering applications, which could be helpful for optimization design of thrust bearing.

\section{Data Availability}

The simulation parameter is referred to Ref. [43].

\section{Conflicts of Interest}

The authors declare that they have no conflicts of interest.

\section{Acknowledgments}

This work is supported by the "Central Public-Interest Scientific Institution Basal Research Fund (No. S202005-01)," "China Postdoctoral Science Foundation (No. 2020M681531)," "Agricultural Science and Technology Innovation Program (Fruits, Vegetables and Tea Harvesting Machinery)," and "National Natural Science Foundation of China (No. 52005230).”

\section{References}

[1] M. T. C. Faria and W. Mendes Miranda, "Pressure dam influence on the performance of gas face seals," Tribology International, vol. 47, pp. 134-141, 2012.

[2] J. Jiang, W. Zhao, X. Peng, and J. Li, "A novel design for discrete surface texture on gas face seals based on a superposed groove model," Tribology International, vol. 147, p. 106269, 2020.

[3] Y. Mao, L. Zeng, and Y. Lu, "Modeling and optimization of cavitation on a textured cylinder surface coupled with the wedge effect," Tribology International, vol. 104, pp. 212-224, 2016.

[4] J. Zhang, D. Zou, N. Ta, Z. Rao, and B. Ding, "A numerical method for solution of the discharge coefficients in externally pressurized gas bearings with inherent orifice restrictors," Tribology International, vol. 125, pp. 156-168, 2018.

[5] S. M. Yao, "Aerostatic and aerodynamic performance of an out-pump spirally grooved thrust bearing: analysis and comparisons to static load experiments," Tribology Transactions, vol. 52, no. 3, pp. 376-388, 2009.

[6] J. C. Hung, C. H. Chang, K. C. Chiu, and S. J. Lee, "Simulationbased fabrication of micro-helical grooves in a hydrodynamic thrust bearing by using ECMM," The International Journal of Advanced Manufacturing Technology, vol. 64, no. 5-8, pp. 813-820, 2013.

[7] L. Zhai, G. Wu, X. Wei, D. Qin, and L. Q. Wang, "Theoretical and experimental analysis for leakage rate and dynamic characteristics of herringbone-grooved liquid seals," Proceedings of the Institution of Mechanical Engineers, Part J, Journal of Engineering Tribology, vol. 229, no. 7, pp. 849-860, 2015.

[8] S. Błasiak and C. Kundera, "A Numerical Analysis of the Grooved Surface Effects on the Thermal Behavior of a NonContacting Face Seal," Procedia Engineering, vol. 39, pp. 315-326, 2012.

[9] Y. Henry, J. Bouyer, and M. Fillon, "An experimental hydrodynamic thrust bearing device and its application to the study of a tapered-land thrust bearing," Journal of Tribology, vol. 136, no. 2, article 021703, 2014.

[10] U. Nishio, K. Somaya, and S. Yoshimoto, "Numerical calculation and experimental verification of static and dynamic characteristics of aerostatic thrust bearings with small feedholes," Tribology International, vol. 44, no. 12, pp. 1790-1795, 2011.

[11] M. Qiu, A. Delic, and B. Raeymaekers, "The effect of texture shape on the load-carrying capacity of gas-lubricated parallel slider bearings," Tribology Letters, vol. 48, no. 3, pp. 315-327, 2012.

[12] X. Q. Zhang, X. L. Wang, R. Liu, and B. Wang, "Influence of temperature on nonlinear dynamic characteristics of spiralgrooved gas-lubricated thrust bearing-rotor systems for microengine," Tribology International, vol. 61, pp. 138-143, 2013. 
[13] S. H. Chang, C. W. Chan, and Y. R. Jeng, "Numerical analysis of discharge coefficients in aerostatic bearings with orifice-type restrictors," Tribology International, vol. 90, pp. 157-163, 2015.

[14] C. Ma, S. Bai, and X. Peng, "Thermo-hydrodynamic characteristics of spiral groove gas face seals operating at low pressure," Tribology International, vol. 95, pp. 44-54, 2016.

[15] P. Novotný, J. Hrabovský, J. Juračka, J. Klíma, and V. Hort, "Effective thrust bearing model for simulations of transient rotor dynamics," International Journal of Mechanical Sciences, vol. 157-158, pp. 374-383, 2019.

[16] Y. Hu and Y. Meng, "Theoretical and experimental study of transient behavior of spiral-groove thrust bearings during start-up," Tribology Transactions, vol. 63, no. 1, pp. 154-172, 2020.

[17] M. S. Kim, J. Shim, J. Kim, D. G. Jang, and S. S. Park, "Multiphysics simulation and experiment of a thrust bearing in scroll compressors," Tribology International, vol. 142, p. 105969, 2020.

[18] W. Xu and J. Yang, "Spiral-grooved gas face seal for steam turbine shroud tip leakage reduction: performance and feasibility analysis,” Tribology International, vol. 98, pp. 242-252, 2016.

[19] S. Ahn, S. Choi, B. Lee, and Y. C. Rhim, "Analyse d'un palier de butee dans un compresseur a spirale de type calandre du cote haut," International Journal of Refrigeration, vol. 69, pp. 251-260, 2016.

[20] J. Zhang, D. Zou, N. Ta, and Z. Rao, "Numerical research of pressure depression in aerostatic thrust bearing with inherent orifice," Tribology International, vol. 123, pp. 385-396, 2018.

[21] M. Fesanghary and M. M. Khonsari, "On the optimum groove shapes for load-carrying capacity enhancement in parallel flat surface bearings: theory and experiment," Tribology International, vol. 67, pp. 254-262, 2013.

[22] M. Ochiai, Y. Sunami, and H. Hashimoto, "Study on angular displacement characteristics on topological optimum design problem of hydrodynamic thrust air bearing," Proceedings of the Institution of Mechanical Engineers, Part J, Journal of Engineering Tribology, vol. 228, no. 9, pp. 997-1007, 2014.

[23] M. Ochiai, H. Sasaki, Y. Sunami, and H. Hashimoto, "Experimental and theoretical verification of impact response on air thrust bearing with topological optimized groove," Tribology Online, vol. 10, no. 2, pp. 115-120, 2015.

[24] W. Wu, Z. Xiong, J. Hu, and S. Yuan, "Application of CFD to model oil-air flow in a grooved two-disc system," International Journal of Heat and Mass Transfer, vol. 91, pp. 293-301, 2015.

[25] C. Ma, S. Bai, and X. Peng, "Thermoelastohydrodynamic characteristics of T-grooves gas face seals," International Journal of Heat and Mass Transfer, vol. 102, pp. 277-286, 2016.

[26] A. Gherca, A. Fatu, M. Hajjam, and P. Maspeyrot, "Influence of surface texturing on the hydrodynamic performance of a thrust bearing operating in steady-state and transient lubrication regime," Tribology International, vol. 102, pp. 305-318, 2016.

[27] S. Jianjun, M. Chenbo, Y. Qiuping, L. Jianhua, Z. Min, and Z. Peiyan, "Numerical analysis on a new pump-out hydrodynamic mechanical seal," Tribology International, vol. 106, pp. 62-70, 2017.

[28] D. Gropper, T. J. Harvey, and L. Wang, "A numerical model for design and optimization of surface textures for tilting pad thrust bearings," Tribology International, vol. 119, pp. 190207, 2018.
[29] X. Lin, R. Wang, S. Zhang, and S. Jiang, "Study on dynamic characteristics for high speed water-lubricated spiral groove thrust bearing considering cavitating effect," Tribology International, vol. 143, p. 106022, 2020.

[30] S. Bai, X. Peng, Y. Meng, and S. Wen, "Modeling of gas thermal effect based on energy equipartition principle," Tribology International, vol. 55, no. 6, pp. 752-761, 2012.

[31] W. Xu and J. Yang, "Explicit solution of gas film pressure for performance calculation of spiral grooved gas seals," Journal of Mechanical Science and Technology, vol. 32, no. 1, pp. 277-282, 2018.

[32] S. Thomas, N. Brunetière, and B. Tournerie, "Numerical modelling of high pressure gas face seals," Journal of Tribology, vol. 128, no. 2, pp. 396-405, 2006.

[33] Y. Wang, H. Yang, J. Wang, Y. Liu, H. Wang, and X. Feng, "Theoretical analyses and field applications of gas-film lubricated mechanical face seals with herringbone spiral grooves," Tribology Transactions, vol. 52, no. 6, pp. 800-806, 2009.

[34] S. Gao, K. Cheng, S. Chen, H. Ding, and H. Fu, "CFD based investigation on influence of orifice chamber shapes for the design of aerostatic thrust bearings at ultra-high speed spindles," Tribology International, vol. 92, pp. 211-221, 2015.

[35] J. Zhang, F. Yu, D. Zou, N. Ta, and Z. Rao, "Comparison of the characteristics of aerostatic journal bearings considering misalignment under pure-static and hybrid condition," Proceedings of the Institution of Mechanical Engineers, Part J: Journal of Engineering Tribology, vol. 233, no. 5, pp. 769781, 2019.

[36] J. Du, G. Zhang, T. Liu, and S. To, "Improvement on load performance of externally pressurized gas journal bearings by opening pressure-equalizing grooves," Tribology International, vol. 73, pp. 156-166, 2014.

[37] M. Xu, Q. Dai, W. Huang, and X. Wang, "Using magnetic fluids to improve the behavior of ball bearings under starved lubrication," Tribology International, vol. 141, article 105950, 2020.

[38] X. Wang, Q. Xu, M. Huang, L. Zhang, and Z. Peng, "Effects of journal rotation and surface waviness on the dynamic performance of aerostatic journal bearings," Tribology International, vol. 112, pp. 1-9, 2017.

[39] M. Qiu, B. R. Minson, and B. Raeymaekers, "The effect of texture shape on the friction coefficient and stiffness of gas- lubricated parallel slider bearings," Tribology International, vol. 67, pp. 278-288, 2013.

[40] F. M. Meng, L. Zhang, Y. Liu, and T. T. Li, "Effect of compound dimple on tribological performances of journal bearing," Tribology International, vol. 91, pp. 99-110, 2015.

[41] X. Wang, Q. Xu, B. Wang, L. Zhang, H. Yang, and Z. Peng, "Effect of surface waviness on the static performance of aerostatic journal bearings," Tribology International, vol. 103, pp. 394-405, 2016.

[42] T. V. V. L. N. Rao, A. M. A. Rani, M. Awang, and F. M. Hashim, "Stability evaluation of three-layered journal bearing with slip/partial slip," Industrial Lubrication and Tribology, vol. 69, no. 3, pp. 334-341, 2017.

[43] R. P. Gabriel, "Fundamentals of spiral groove noncontacting face seals," Lubrication Engineering, vol. 50, no. 3, pp. 214224, 1994.

[44] M. Deligant, P. Podevin, and G. Descombes, "CFD model for turbocharger journal bearing performances," Applied Thermal Engineering, vol. 31, no. 5, pp. 811-819, 2011. 